\title{
Scaling of Heat Transport near Onset in Rapidly Rotating Convection
}

\author{
Robert E. Ecke
}

Center for Nonlinear Studies, Los Alamos National Laboratory, Los Alamos, NM 87545, USA

\begin{abstract}
We consider the scaling of heat transport in the geostrophic regime of rotating Rayleigh-Bénard convection near onset for small Ekman number $E k$ from the perspective of weakly nonlinear theory. We show that available heat transport data from numerical simulation [1] for $E k<10^{-5}$ for $\operatorname{Pr}=1$ are consistent with weakly nonlinear theory for $\epsilon=R a / R a_{c}-1<1$. In particular, we show that the numerical data are consistent with $N u-1=a \epsilon+b \epsilon^{2}$ with $a \approx 2$ and $b \approx 3$ with weak dependence of the coefficients on $E k$. The coefficient $a$ is consistent with calculations of weakly nonlinear theory and with experimental data at much higher $E k$. The positive sign of $b$ is also suggested by those experimental data. The magnitude and trend of the numerical data for larger $R a$ are consistent with experimental data with similar $\operatorname{Pr} \sim 1$. The steep scaling of $N u \sim\left(R a / R a_{c}\right)^{3}$ noted elsewhere for $R a / R a_{c}<2$ is shown to be an artifact of being close to onset where the effective power-law slope depends sensitively on the magnitude of the coefficients $a$ and $b$.
\end{abstract}

The regime of geostrophic rotating Rayleigh-Bénard convection develops for rapidly rotating conditions where Coriolis forces dominate buoyancy [1, 2, 3, 4] and the bulk flow is in geostrophic balance, i.e., lateral pressure gradients are balanced by the Coriolis force. The competition of buoyancy and rotation is represented by the convective Rossby number $R o=(R a /(\operatorname{Pr} T a))^{1 / 2}=(R a / P r)^{1 / 2} E k$ where $R a=g \alpha H^{3} \Delta T /(\nu \kappa), \operatorname{Pr}=\nu / \kappa$, $E k=\nu /\left(2 \Omega H^{2}\right)$, and $T a=E k^{-2}$ with gravity $g$, thermal expansion coefficient $\alpha$, cell height $H$, vertical temperature difference $\Delta T$, kinematic viscosity $\nu$, and thermal diffusivity $\kappa$. The geostrophic regime occurs for $R o \quad<1$ but subject to the

Preprint submitted to Physics Letters A limit that bulk convection is suppressed for $R a<R a_{c}=A E k^{-4 / 3}$ where $A$ is weakly (logarithmically) dependent on $E k$ with an asymptotic value of $A=8.7$ for $E k \rightarrow 0$ [5]. It is convenient in this context to consider both the scaled Rayleigh number $\hat{R} a=R a / R a_{c}$ and the normalized distance from onset $\epsilon=R a / R a_{c}-1$; they are related: $\epsilon=\hat{R} a-1$.

The lower limit of geostrophic rotating convection is set by the condition $R o_{c}=$ $(A / P r)^{1 / 2} E k^{1 / 3}$ at which $N u=1$. The upper limit at constant $E k$ is set by the crossover to boundary layer dominated convection where $N u=B R a^{\beta}$ with $2 / 7<\beta<1 / 3$. The heat transport in this latter regime has been rather well studied $[6,7,8,9,10]$ with regimes of en-

June 25, 2015 

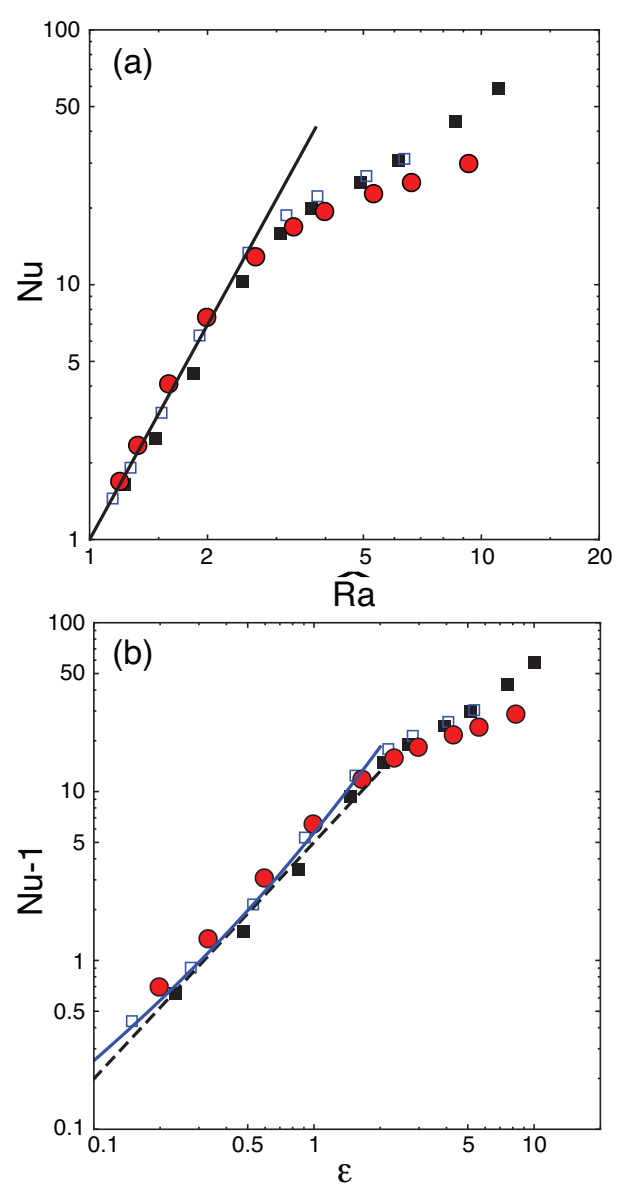

Figure 1: (a) $N u$ vs $\hat{R} a, N u=\hat{R} a^{2.8}$ (solid line); (b) $N u-1$ vs $\epsilon, N u-1=2.2 \epsilon+3.5 \epsilon^{2}$ (solid line), $N u-$ $1=5 \epsilon^{1.4}$ (dashed line). $\operatorname{Pr}=1$ and $E k: 10^{-7}$ (solid square, black), $10^{-6}$ (open square, blue), $10^{-5}$ (solid circle, red).

hancement of $N u$ relative to non-rotating turbulent convection at moderate $\operatorname{Ra}$ and $\operatorname{Pr}>$ 1 owing to Ekman suction/pumping of the boundary layer. The enhancement vanishes for large enough $R a$ or low enough $\operatorname{Pr}$ [10]. The crossover to this regime is consistent with the condition that the thermal boundary layer thickness $\delta_{T}=H /(2 N u)$ is of order the Ekman layer thickness $\delta_{E}=H E k^{1 / 2}$ [9], leading to the relationship $R a E k^{1 /(2 \beta)} \approx c$. The range of geostrophic rotating convection is then $1<\hat{R} a<c E k^{-1 /(2 \beta)+4 / 3}$. If we take $\beta=2 / 7$ consistent with experimental re- sults in water at modest $R a[8,9]$, we get an upper range proportional to $E k^{-5 / 12}$ whereas with $\beta=0.31$ as in convection in helium gas [11] one has $E k^{-2 / 7}$ scaling. In both cases, the geostrophic regime grows slowly with decreasing $E k$ and requires small $E k$ to obtain even modest ranges of $\hat{R} a$ and $N u$ given by $1<N u<B c^{1 / 2} E k^{-1 / 2}$. Recent measurements in rotating cryogenic helium convection [4] allowed determination of the upper range of $N u$ but data close to onset at small $E k$ are hard to obtain. In this context, numerical simulations can provide a complementary approach and access to states near the onset of convection that are difficult to obtain in experiments when $E k$ is small.

Recently, Stellmach et al [1] reported on high-resolution numerical simulations of rotating Rayleigh-Bénard convection at very small Ekman number in the range $10^{-7}<E k<$ $10^{-5}$ and for two values of $\operatorname{Pr}: 1$ and 7 . Their analysis of the resulting heat transport $N u$ as a function of $\tilde{R} a=R a E k^{4 / 3}=A \hat{R} a$ suggested the relationship $N u \sim \tilde{R} a^{3}$ and a significant enhancement of $N u$ for no-slip boundary conditions relative to that for free-slip boundary conditions [1]. The correspondence between the $R a^{3}$ power law and marginal stability arguments leading to this cubic scaling [12] were noted. These data for $\mathrm{Pr}=1$ are plotted in Fig. 1(a) with a power law slope $N u \sim \hat{R} a^{2.8}$ indicated by the solid line with only weak dependence of the prefactor on $E k$. How can we understand this large power-law scaling and its significance relative to the physical mechanisms discussed in [1]? 

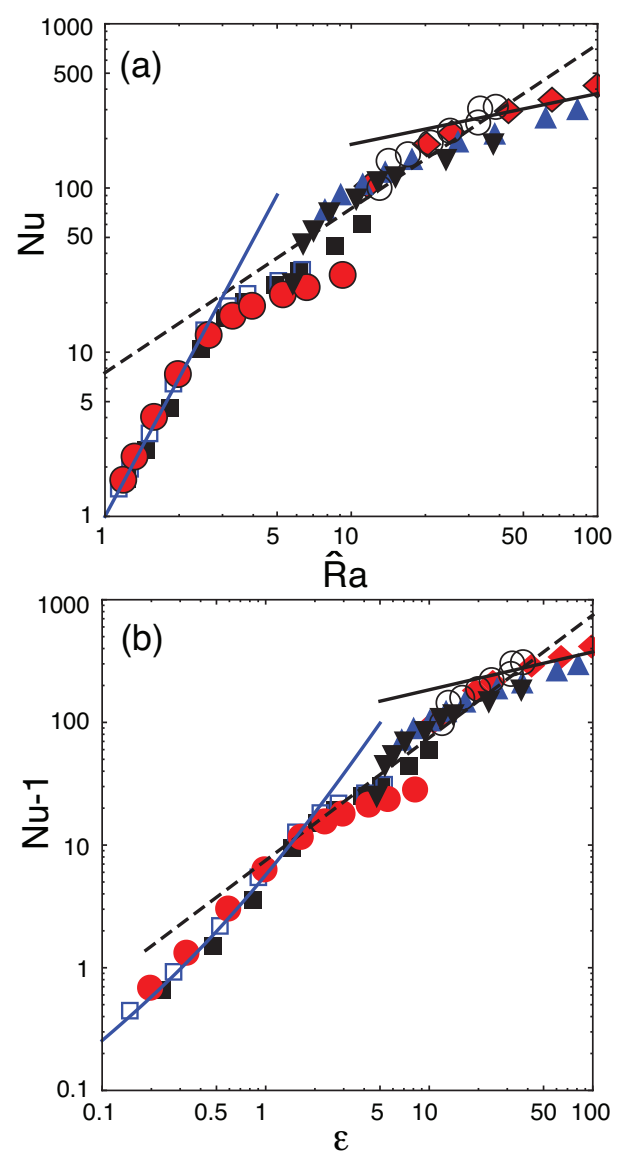

Figure 2: (a) $N u$ vs $\hat{R} a: N u=\hat{R} a^{2.8}$ (solid line), $N u \sim \hat{R} a$ (dashed line), $N u \sim \hat{R} a^{0.31}$ (solid line). (b) $N u-1$ vs $\epsilon: N u-1=2.2 \epsilon+3.5 \epsilon^{2}$ (solid line), $N u-1 \sim \epsilon$ (dashed line), $N u-1 \sim \epsilon^{0.31}$ (solid line). Numerical simulations [1]: $\operatorname{Pr}=1$ and $E k: 10^{-7}$ (solid square, black), $10^{-6}$ (open square, blue), $10^{-5}$ (solid circle, red). Experimental data [4] for $\mathrm{Pr}=0.7$ and $E k: 2.1 \times 10^{-7}$ (solid down triangles, black), $E k$ : $3.1 \times 10^{-7}$ (solid diamonds, red), $5.9 \times 10^{-7}$ (solid up triangles, blue), $1.1 \times 10^{-6}$ (open circles, black).

First, we note that the range over which the approximate cubic power law is observed has $1<\hat{R} a<2$ corresponding to a range $0.1<$ $\epsilon<1$. Over this range close to the onset of convection, one might consider an alternative representation of the data suggested by weakly nonlinear theory: $N u-1=a \epsilon+b \epsilon^{2}+\ldots$ (see, for example, [13]). There are many limitations to this approach, the most pronounced being that it is only strictly valid for $\epsilon<<1$ but also, for the rotating convection problem, the weakly nonlinear state is complex owing to intrinsic time dependence arising from the Küppers-Lortz instability [14]. Nevertheless, let's see how well a fit to a weakly nonlinear expansion describes the data. In Fig. 1(b), we show the data represented as $N u-1$ versus $\epsilon$ in which there is a relatively straight section of data from $\epsilon \approx 0.1$ up to $\epsilon \approx 2$. A simple power law $N u=5 \epsilon^{1.4}$ describes the data adequately as indicated by the dashed line. On the other hand, a weakly nonlinear form $N u-1=2.2 \epsilon+3.5 \epsilon^{2}$ (variations of about $10 \%$ in $a$ and $30 \%$ in $b$ yield similar results) also fits the data well, better accounting for the slight upward curvature in the data for the smaller $E k$ values.

A comparison with predictions of nonlinear theory for rapidly rotating convection [14, $15,5]$ suggests a value for $a$ close to 2 in the range $10^{-7}<E k<10^{-5}$ (asymptotically precisely 2 as $E k \rightarrow 0$ [5]). This predicted value is within $10 \%$ of the rough $a \approx 2.2$ consistent with the numerical data shown in Fig. 1(b); the data are not extensive enough to be more precise. Further, experimental data from cryogenic helium data with $\operatorname{Pr}=0.55$ at $E k=0.9^{-3}[16]$ yields $a=2.08$ (the prediction from weakly nonlinear theory [15] is 2.07). Although for large $E k>10^{-3}$, the coefficient $b$ is typically negative (see Table 3 of [16]), it seems to take on a positive value for $E k<10^{-3}: b=0.20$ for $E k>1.1 \times 10^{-3}$ and $b=0.38$ for $E k=0.9^{-3}$. This trend is consistent with the positive coefficient $b=3.5$ in the approximation to the data in Fig. 1 (b) although the large magnitude is perhaps surprising. An extension to next higher order in $\epsilon$ in weakly nonlinear theory would be helpful in resolving the observed value of $b$ although the current data seem consist with predictions of weakly nonlinear theory and extrapolation of data with similar $\operatorname{Pr}$. A final point here 
is that the apparent power-law exponent of a weakly-nonlinear expansion can be written as $\alpha(x)=d \log N u / d \log x=x(a+2 b(x-$ $1)) /\left(1+a(x-1)+b(x-1)^{2}\right)$ where $x=\hat{R} a$. One sees that the apparent power law exponent is very sensitive to the coefficient magnitude with, for example, $\alpha(1)=a$ at onset. For intermediate $x<2$, other values are obtained such that if we use the values $a$ and $b$ consistent with the numerical data, we obtain $\alpha(1.5) \approx 3$.

It is useful to compare the numerical results [1] for $\operatorname{Pr}=1$ with the experimental results for similar $\operatorname{Pr}=0.7$ using cryogenic helium [4]. We show this comparison in Figs. 2 (a) \& (b), plotting the data in both representations. Although the data barely overlap at intermediate values of $\hat{R} a$, they appear reasonably consistent given that the experimental data are more uncertain at lower values whereas the opposite is the case for the numerical simulations. The data in the intermediate range for both plots is consistent with $N u \sim \hat{R} a$. The solid line at larger $R a$ indicates the $R a^{1 / 3}$ power law in the boundarylayer controlled regime. Overall, the agreement is satisfactory but better quality experimental data at smaller $\hat{R} a$ and numerical data at higher $\hat{R} a$ will be needed for a more detailed comparison and a convincing determination of the behavior of heat transport in the geostrophic range of rapidly rotating convection.

The analysis above suggests a quite large region of convergence of the weakly nonlinear form up to values $\epsilon \approx 2$. This is a bit surprising so let's look more carefully at the data, remembering that log-log plots can hide features or blemishes. In Fig. 3(a), we plot, for $\operatorname{Pr}=1,(N u-1) / \epsilon$ versus $\epsilon$ on a linear-linear scale to accentuate deviations from a weakly nonlinear form.
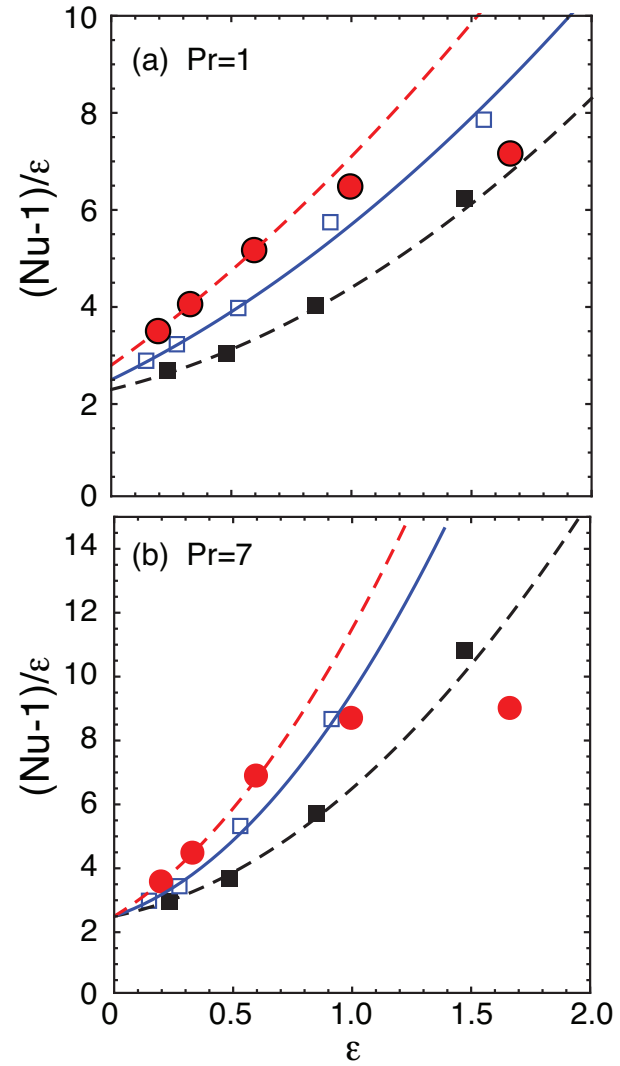

Figure 3: $(N u-1) / \epsilon$ vs $\epsilon . E k: 10^{-7}$ (solid square, black), $10^{-6}$ (open square, blue), $10^{-5}$ (solid circle, red). (a) $\operatorname{Pr}=1$. Lines are quadratic fits with coefficients: $\{2.3,1.2,0.9\},\{2.5,2.4,0.8\},\{2.8,3.5,0.8\}$. (b) $\operatorname{Pr}=7$. Lines are quadratic fits with coefficients: $\{2.5$, $1.5,2.5\},\{2.5,2.5,4.5\},\{2.5,4.5,4.5\}$

The intercept is the coefficient $a$, the initial linear slope is the coefficient $b$, and there is significant curvature in the data that requires a cubic correction $c$. In this representation, we have $2.3<a<2.8,1.2<b<3.5$ and $c \approx 0.8$. For comparison, we show in Fig. 3(b) the same plot for numerical data with $\operatorname{Pr}=7$ [1]. The coefficient $a \approx 2.5$ is about the same for the different $\mathrm{Pr}$ although the coefficients $1.8<b<4.5$ and $2.5<c<4.5$ are significantly larger. One gets the impression that there are deviations from weakly nonlinear theory at smaller $\epsilon$ for $\operatorname{Pr}=7$. As an estimate of the range of $\epsilon$ over which one seems to have a convergent expansion, we can take the 
criterion that $(b / a) \epsilon<\gamma$ (and similarly that $(c / b) \epsilon<\gamma)$ with $\gamma<1$. Choosing $\gamma=0.2$ yields a convergence range of weakly nonlinear theory for the $\mathrm{Pr}=1$ data of between 0.2 and 0.5 . Note that the range seems to get larger for smaller $E k$. For the $\operatorname{Pr}=7$ data, we have a smaller $\epsilon$ convergence range of between 0.1 and 0.3 , consistent with ones impression from Fig. 3. For both data sets it would be highly useful to obtain more points for $\epsilon<1$ to better constrain these fits; it is certainly a stretch to be fitting a cubic expansion to only three points.

From this representation of the data, what can we conclude from the consistency of the scaling of $N u-1$ with $\epsilon$ for $\epsilon<1$ with weakly nonlinear theory? Certainly, the regime of geostrophic turbulence at higher $\hat{R} a$ [3] is far beyond weakly nonlinear theory and many of the interesting features of geostrophic Taylor columns [2] also may not be captured. Further, the Ekman pumping mechanism demonstrated in the numerical simulations of [1] may be intimately related to the large magnitude of the weakly nonlinear coefficients. Thus, exploring the crossover from a weakly nonlinear regime to the strongly nonlinear states emerging at $\epsilon \sim 1$ would appear fruitful.

In summary, the scaling of heat transport in the geostrophic range of rotating RayleighBénard convection near the linear onset computed from numerical simulation [1] is consistent with weakly nonlinear theory which predicts that $N u-1=a \epsilon+b \epsilon^{2}+\ldots$ for small $\epsilon$. It is seen that the large power-law dependence $N u \sim \hat{R} a^{3}$ arises from the magnitude of the weakly nonlinear coefficients, in particular $a \approx 2$ which is consistent with values from both calculation and experimental data at larger $E k$. Since Ekman pumping is turning on over the region of interest, it would be of value to understand how the weakly non- linear state is reflecting that increase and also how the flow develops the interesting features of Taylor columns and geostrophic turbulence as nonlinearity kicks in.

We thank S. Stellmach for providing the numerical data used in this paper. We acknowledge important conversations with S. Stellmach, J. Aurnou, K. Julien, and I. Christov. This work was funded by the LDRD Program at Los Alamos National Laboratory by the National Nuclear Security Administration of the U.S. Department of Energy under Contract No. DE-AC52-06NA25396.

[1] S. Stellmach, M. Lischper, K. Julien, G. Vasil, J. S. Cheng, A. Ribeiro, E. M. King, and J. M. Aurnou, Phys. Rev. Lett. 113, 254501 (2014).

[2] I. Grooms, K. Julien, J. B. Weiss, and E. Knobloch, Phys. Rev. Lett. 104, 224501 (2010).

[3] K. Julien, E. Knobloch, A. M. Rubio, and G. M. Vasil, Phys. Rev. Lett. 109, 254503 (2012).

[4] R. E. Ecke and J. J. Niemela, Physical Review Letters 113, 114301 (2014).

[5] J. Dawes, J. Fluid Mech. 428, 61 (2001).

[6] H. T. Rossby, J. Fluid Mech. 36, 309 (1969).

[7] F. Zhong, R. E. Ecke, and V. Steinberg, J. Fluid Mech. 249, 135 (1993).

[8] Y. Liu and R. Ecke, Phys. Rev. Lett. 79, 2257 (1997).

[9] E. King, S. Stellmach, J. Noir, U. Hansen, and J. Aurnou, Nature 457, 301 (2009).

[10] J.-Q. Zhong, R. Stevens, H. Clercx, R. Verzicco, D. Lohse, and G. Ahlers, Phys. Rev. Lett. 102, 044502 (2009).

[11] J. J. Niemela, L. Skrbek, K. R. Sreenivasan, and R. J. Donnelly, Nature 404, 837 (2000).

[12] E. King, S. Stellmach, and J. Aurnou, J. Fluid Mech. 691, 568 (2012).

[13] M. Cross and P. Hohenberg, Reviews of Modern Physics 65, 851 (1993).

[14] T. Clune and E. Knobloch, Physical Review E 47, 2536 (1993).

[15] A. Bassom and K. Zhang, Geophys. Astrophys. Fluid Dyn. 76, 223 (1994).

[16] J. M. Pfotenhauer, P. Lucas, and R. J. Donnelly, J. Fluid Mech. 145, 239 (1984). 